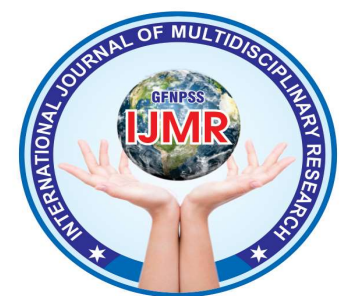

\title{
EVIDENCE BASED PRACTICE IN NURSING
}

\section{PROF. RAJVEER SINGH}

\section{Principal \& HOD Medical Surgical Nursing,}

Saket Nursing College, Jaipur

\section{Corresponding Email:creativeraj singh@yahoo.com}

Evidence-based practice is a method by which healthcare providers or nurses across the healthcare professions review and assess the most contemporary, highest-quality research quest to notify their delivery of healthcare.

Keyword: Evidence based practice

\begin{tabular}{ccc}
\hline Received & Accepted & Available online \\
\hline $31 / 07 / 2021$ & $04 / 08 / 2021$ & $05 / 08 / 2021$ \\
\hline
\end{tabular}

\section{EVIDENCE BASED NURSING (EBN)}

EVB ensure that provided care is appropriate and cost effective so clients will have better outcomes.

Sources of evidence- based nursing practice are:

- Expert opinion

- Tradition (the way we always do it)

- Trial and error

- Borrowed from other disciplines

- Based on scientific research

\section{A's of evidence-based practice}

- We therefore advocate to be more explicit and aim to clarify the distinction between EBP for the individual patient and for a group of patients or caregivers by discussing the following five steps: ask, acquire, appraise, apply and assess TYPES OF EVIDENCE: -The Canadian Institutes for Health Research (2009) created a hierarchy of quantitative evidence

- Systematic reviews of randomized controlled trials;

- Randomized controlled trials;

- systematic reviews of nonrandomized or cohort studies;

- Cohort studies;

- Case control studies;

- Case Series;

- Case Report;

- Ideas, editorials, opinions;

- Animal research; and

- Invitro (test tube) research. 


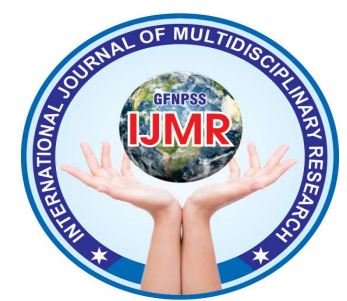

EBM requires integration of three major components for medical decision making: 1) the best external evidence, 2) individual practitioner's clinical expertise, and 3) patients' preference.

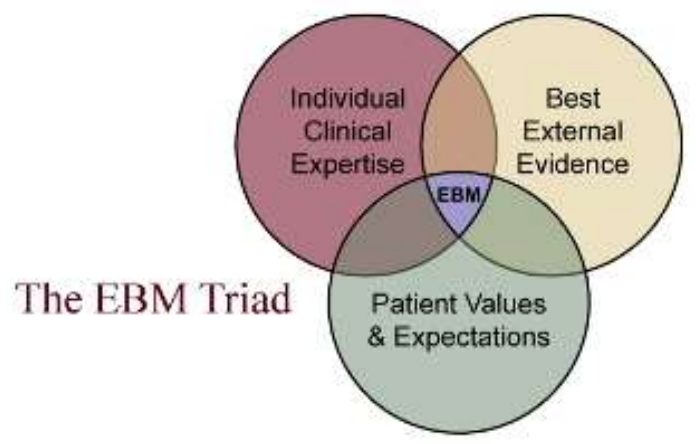

\section{Need of EBP:-}

EBP is important because it aims to provide the most effective care that is available, with the aim of improving patient outcomes. Patients expect to receive the most effective care based on the best available evidence. EBP promotes an attitude of inquiry in health professionals and starts us thinking about: Why am I doing this in this way? Is there evidence that can guide me to do this in a more effective way? As health professionals, part of providing a professional service is ensuring that our practice is informed by the best available evidence. EBP also plays a role in ensuring that finite health resources are used wisely and that relevant evidence is considered when decisions are made about funding health services. ${ }^{4}$

Evidence-based practice demands changes in education of students, more practice-relevant research, and closer working relationships between clinicians and researchers. Evidencebased practice also provides opportunities for nursing care to be more individualized, more effective, streamlined, and dynamic, and to maximize effects of clinical judgment.

It provides useful insight into evidence-backed methods in nearly every possible sector of nursing, such as direct patient care, workflow issues, infection control, supply management, charting, central line care, etc.

\section{FIVE STEPS TO TRANSITION TO} EVIDENCE-BASED MEDICINE: -

Moving entire organizations over to an evidencebased model of care presents a few challenges. First, adopting evidence-based medicine as a new standard of practice requires clinicians to change how they were taught to diagnose and treat patients (i.e., the traditional craftsman approach taught during residency or nursing school). Second, providers are already simply trying to keep up with their workloads to treat patients the best they know how. Introducing a new model of care delivery-and learning how to effectively use it - takes time.

There are five steps 


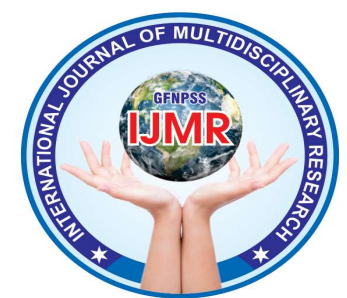

busy clinicians can take to incorporate evidencebased medicine into their practices:

Step \#1: Ask a clinical question to identify a key problem:

The first step to moving to an evidence-based model of care is to form teams of clinicians whose role is to ask questions to identify the critical few problems that need to be addressed. Examples of major problems most healthcare organizations need to address include improving throughput and reducing readmissions.

It's important to identify a high-priority problem that offers a significant return on investment. Once clinicians see large gains, they become engaged and excited about moving to an improved model of care. Women's services, ischemic heart care, and surgical departments are examples of areas that typically offer opportunities for major improvements. Clinicians can ask several questions to help identify problems:

- Why are we practicing this way? Clinicians naturally practice a certain way because that's how they were taught. Yet with new medical discoveries coming out seemingly every day, what was once a best practice may not be anymore.

- Are we adhering to best practices? It's important to ask this question to determine if current practices are based on today's most researched processes and practices that lead to the best possible outcomes.

- Can we produce better outcomes with consistency? Care should always be provided based on the best available evidence. This reduces inconsistent outcomes, which improves quality and reduces costs.

\section{Step 2: Acquire the best evidence (medical knowledge) possible.}

There are many different sources of evidencefrom the knowledge clinicians gain from treating their patient populations to new research being discovered from highly organized randomized controlled trials (RCTs). But not all of this knowledge represents high-quality evidence that provides the best insight for patient care. To help clinicians compare the quality between the various sources of evidence, David Sackett, MD, popularized the evidence-based medicine pyramid. Within in this pyramid, evidence in the top level is the strongest because it underwent a systematic review process and meta-analysis. Evidence in the lowest level is the weakest because it is primarily based on opinions and small sample sizes, which increases room for error. Transitioning to an evidence-based model of care means encouraging clinicians to use the evidence found in the upper levels of the pyramid 


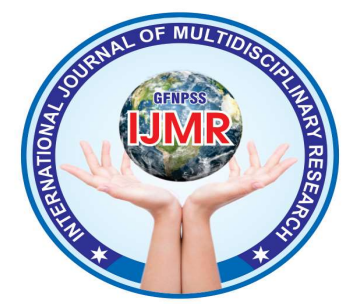

to see better outcomes with their patients. As stated in the abstract Evidence-based nursing practice: why is it important?, "Practices based on research findings are more likely to result in the desired patient outcomes across various settings and geographic locations."

\section{Step 3: Appraise the evidence (make sure it's} applicable to the population and the question asked).

There's good evidence (e.g., from RCTs) and there's bad evidence (e.g., from personal experience). Then there's evidence that falls in the gray area-neither clearly defined as good nor bad. It can be difficult for clinicians to know whether to use evidence in the gray area.

To determine the evidence's validity, a team of clinicians with several years of evidence appraisal experience should review the knowledge in question. The team can then determine if the evidence is valid (i.e., accurate) and applicable (i.e., useful for the situation or population being considered).

\section{Step 4: Apply the evidence to daily clinical practice.}

If the evidence passes the appraisal step and adds value to the practice of medicine, then clinicians can incorporate the new knowledge into their daily clinical practice. It's important to note that evidence-based medicine doesn't replace a clinician's expertise or judgment. Its purpose is to enhance their ability to make better care decisions based on the needs and preferences of the patient. Since not all clinicians will understand the value of adopting a new standard of care, it's essential to communicate the change and the benefit it adds to patient care. Clinicians will also appreciate if the addition of the new knowledge can be built into their current flow of work and not add extra work to their already busy schedules. Done well, this could improve workflow rather than detract from it.

\section{Step 5: Assess your performance.}

Last, assess any improvements to performance or outcomes the new evidence provides. This can be accomplished by establishing a baseline and then measuring improvements. This is an ongoing process - reassessing and re-measuring any gains or losses should be part of an ongoing cycle to ensure the best outcomes.

\section{EVIDENCE-BASED MEDICINE: KEY \\ TAKEAWAYS}

\section{Three Key Components of Evidence-Based}

\section{Medicine}

1. Research-based evidence.

2. Clinical expertise (i.e., the clinician's accumulated experience, knowledge, and clinical skills).

3. Patients' values and preferences. 


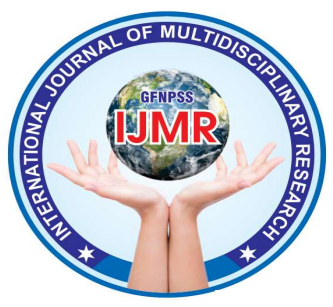

Five Ways Evidence-Based Medicine Adds Value to Health Systems

When evaluating EBP nursing research studies, focus on these four criteria:

1. Helps clinicians stay current on standardized, evidence-based protocols.

2. Uses near real-time data to make care decisions.

3. Improves transparency, accountability, and value.

4. Improves quality of care.

5. Improves outcomes.

Five Steps to Transition to Evidence-Based Medicine

1. Ask a clinical question.

2. Acquire the best evidence possible.

3. Appraise the evidence (make sure it's applicable to the population and the question asked).

4. Apply the evidence.

5. Assess your performance.

Has your practice incorporated evidence-based medicine? If so, what has your experience been? If not, would you like to see this approach to care adopted at your organization?

\section{The 5 Steps of Evidence-Based Medicine}

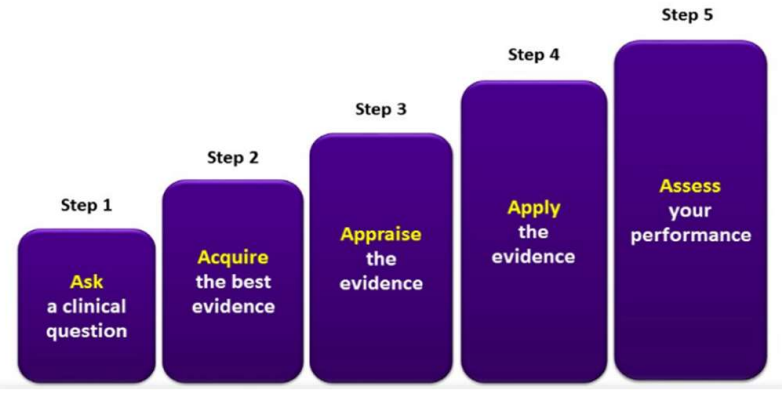

- Infection Control

- Oxygen Use in Patients with COPD

- Measuring Blood Pressure Noninvasively in Children.

- Intravenous Catheter Size and Blood Administration. 


\section{Infection Control}

- The last thing a patient wants when going to a hospital for treatment is a hospital-acquired infection. Nurses play a key role in helping to prevent illness before it happens by adhering to evidence-based infection-control policies. This includes keeping the healthcare environment clean, wearing personal protective clothing, using barrier precautions and practicing correct handwashing. Although nurses are busy with many responsibilities, the time it takes to control infection is well worth the effort.

\section{Oxygen Use in Patients with COPD}

- For patient health and safety, it is essential that nurses follow evidence-based practice in nursing when it comes to giving oxygen to patients with COPD. Despite the belief by some that providing oxygen to these patients can create serious issues such as hypercarbia, acidosis or even death, the evidence-based protocol is to provide oxygen to COPD patients. This practice can help prevent hypoxia and organ failure. Giving oxygen, which is the correct treatment based on the evidence, can enhance COPD patients' quality of life and help them live longer.

\section{Measuring Blood Pressure}

Noninvasively in Children
- Nurses should measure blood pressure according to evidence-based practice because accurate measurements are an essential part of effective treatment. Measuring blood pressure in children is a different procedure than it is for adults. Measuring children's blood pressure involves the auscultatory method, then comparing the measurement against data gathered with the oscillometric method.

\section{Intravenous Catheter Size and Blood}

\section{Administration}

- Nurses should follow EBP when using intravenous catheters to administer blood for packed red blood cell transfusions (PRBC). The protocol indicates that nurses should use a smaller-gauge catheter, which increases patient comfort.

\section{STEPS OF EVIDENCE-BASED NURSING}

\section{- Ask a question}

Converting the need for information (about prevention, diagnosis, prognosis, therapy, causation, etc) into an answerable question

- Find information/evidence to answer question Tracking down the best evidence with which to answer that question

- Critically appraise the information/ evidence Critically appraising that evidence for its validity (closeness to the truth), impact (size of the effect), and applicability (usefulness in our clinical practice) 


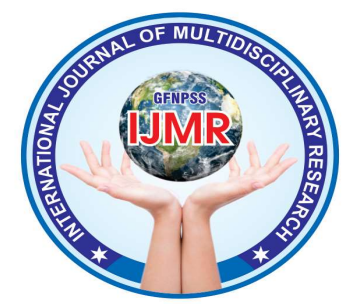

- Integrate appraised evidence with own clinical expertise and patient's preferences Integrating the critical appraisal with our clinical expertise and with our patient's unique biology, values and circumstances

\section{- Evaluate}

Evaluating our effectiveness and efficiency in executing Steps 1-4 and seeking ways to improve them both for next time

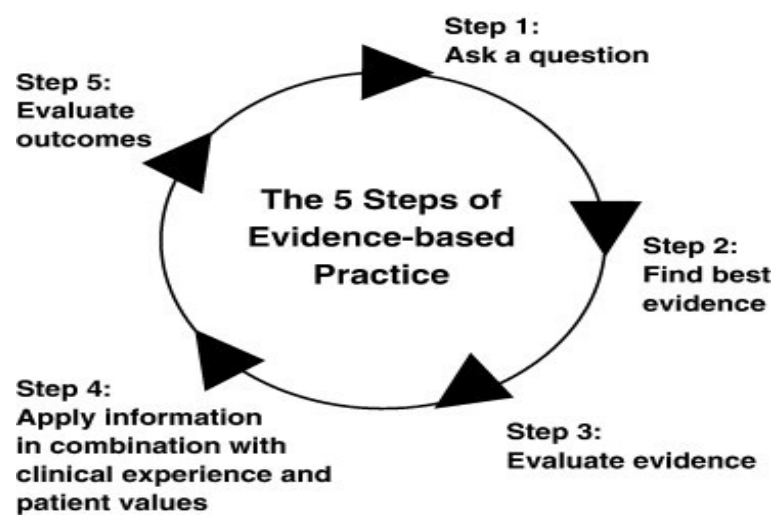

BARRIERS OF EVIDENCE-BASED NURSING CARE

A study conducted in Iran 2014 found that 57\% of barriers to implementation of EBP are related to individual aspects.

The three individual barriers most often encountered are lack of time to read literature, lack of ability to work with computer, and insufficient proficiency in English language.
- Lack of the knowledge needed to interpret statistical analyses;

- Lack of interest;

- Lack of confidence in critical appraisal skills;

- Lack of knowledge and skills to confidently conduct computer-based literature searches and utilize the research process;

- Nurses feeling overwhelmed by the volume of evidence (Wells, Free \& Adam, 2007);

- Nurses' perceptions that they lack the authority and cooperation to change patient care procedures;

- Negative beliefs, attitudes and values; and

- Educational preparation.

*Except where otherwise indicated, all items in the above list are from Bradshaw, 2010.

\section{ORGANIZATIONAL CHARACTERISTICS:}

- Limited or lack of time;

- Heavy patient workloads;

- Inadequate staffing;

- Limited access to resources;

- Lack of support from nurse managers;

- Different goals for practice between administrators and staff nurses (Van Patter Gale $\&$ Schaffer, 2009); and

- Lack of evidence-based practice mentors in health-care systems (Bertulis, 2008).

\section{NURSE CHARACTERISTICS: *}




\section{REFERENCE:}

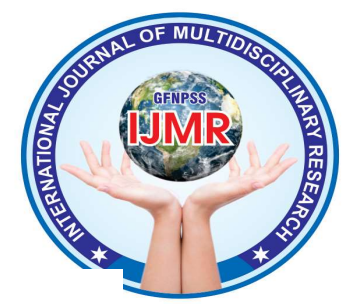

1. FGCU Library, "Evidence Based Practice (NUR 4169): What Is EBP?” Last updated June 22, 2020: https://fgcu.libguides.com/EBP [ $\hookleftarrow]$

2. Jones, K. (2010). Rating the level, quality, and strength of the research evidence. Journal of Nursing Quality, 25, 304-312

3. Guyatt, G.H., Haynes, R.B., Jaeschke, R.Z., \& Cook, D.J. (2000). Users' guides to the medical literature: XXV. evidence-based medicine: principles for applying the users' guides to patient care. JAMA, 284, 12901296. doi: http://dx.doi.org/10.1001/jama.284.10.1290

4. https://online.lasalle.edu/articles/rnbsn/impor tance-of-evidence-based-practice.aspx
5. https://www.healthcatalyst.com/acceleratedpractices-program-healthcare-outcomesimprovement

6. https://degree.astate.edu/articles/nursing/exa mples-of-evidence-based-practice-innursing.aspx

7. Evidence-based medicine : how to practice and teach EBM. - 3rd ed. / Sharon E. Strauss [et al.]. - Edinburgh ; New York :

Elsevier/Churchill Livingstone, 2005

8. Mohammad Khammarnia, ${ }^{1}$ Mahsa Haj Mohammadi, Barriers to Implementation of Evidence Based Practice in Zahedan Teaching Hospitals, Nurs Res Pract. 2015; 2015: 357140 . doi: 10.1155/2015/357140 